\title{
Programa en Autoeficacia, Afecto Positivo y Autoatribución para Promover Conductas Alimentarias Saludables en Estudiantes de Secundaria
}

\author{
Program in Self-efficacy, Positive affect and Self-attribution to promote Healthy Eating \\ Behaviors in Secondary Students
}

\author{
Anahi Gaspar-Pérez ${ }^{a}$, Rebeca M.E. Guzmán-Saldaña ${ }^{b}$, Lilian E. Bosques-Brugada ${ }^{c}$, Silvia \\ Platas-Acevedo ${ }^{d}$, Rosalía Vázquez-Arévalo ${ }^{e}$, Rosalinda Guadarrama-Guadarrama ${ }^{f}$
}

\begin{abstract}
:
The objective of this study was to determine the effectiveness of a program based on universal prevention to modify levels of selfefficacy, positive affect and self-attribution in first and second year secondary school students of a public secondary school in the municipality of Mineral de la Reforma, Hidalgo. The study was quasi-experimental with four groups: two experimental groups and two waiting groups; with pre and post evaluation, participants were randomly assigned according to the grade and group to which they belonged to the experimental groups; and the same to the waiting groups. The total sample was $\mathrm{N}=129$ adolescents, of which $n=63$ belonged to the experimental groups and $n=66$ to the waiting groups; $n=68(53 \%)$ are women and $n=61(47 \%)$ men, in an age range of 12 to 15 years $(\mu=13$ years, SD = .79). A battery of instruments was applied consisting of: Inventory of Self-efficacy for weight control, Scale of Positive and Negative Affection (PANAS) and Self-attribution Inventory for secondary school adolescents. The intervention consisted of 3 sessions of 50 minutes each, which addressed aspects associated with factors promoting Healthy Eating Behaviors. For the statistical analysis, Student's t was used for related samples, the results indicated statistically significant differences in the level of negative self-attribution in the first year experimental group before and after the intervention, $t$ $(32)=2.21, p<0.03$, significantly reducing negative self-attribution $(\mu=2.15)$ after the intervention $(\mu=1.98)$
\end{abstract}

\section{Keywords:}

Universal Prevention, Healthy Eating Behaviors, Self-efficacy, Positive Affect, Self-Attribution.

\begin{abstract}
Resumen:
El objetivo de este estudio fue determinar la efectividad de un programa basado en prevención universal para modificar niveles de autoeficacia, afecto positivo y autoatribución en escolares de educación secundaria de primer y segundo año de una secundaria pública del municipio de Mineral de la Reforma, Hidalgo. El estudio fue cuasi experimental con cuatro grupos: dos grupos experimentales y dos grupos en fase de espera; con pre y post evaluación, de manera aleatoria se asignaron a los participantes de acuerdo al grado y grupo al que pertenecían a los grupos experimentales; y lo mismo a los grupos en fase de espera. La muestra total fue de $\mathrm{N}=129$ adolescentes, de los cuales $\mathrm{n}=63$ pertenecieron a los grupos experimentales y $\mathrm{n}=66$ a los grupos en fase de espera; $\mathrm{n}=68(53 \%)$ son mujeres y $\mathrm{n}=61(47 \%)$ hombres, en un rango de edad de 12 a 15 años $(\mu=13$ años, DE=.79). Se aplicó una batería de instrumentos conformada por: Inventario de Autoeficacia para el control de peso, Escala de Afecto Positivo y Negativo (PANAS) e Inventario de Autoatribución para adolescentes de secundaria. La intervención consistió en 3 sesiones de 50 minutos cada una, que abordaron aspectos asociados con factores de promoción de Conductas Alimentarias Saludables. Para el análisis estadístico se utilizó la $t$ de Student para muestras relacionadas, los resultados indicaron diferencias estadísticamente significativas en el nivel de autoatribución negativa en el grupo experimental de primer año antes y después de la intervención, $\mathrm{t}(32)=2.21, p<$ 0.03 , disminuyendo significativamente la autoatribución negativa $(\mu=2.15)$ después de la intervención $(\mu=1.98)$.
\end{abstract}

\section{Palabras Clave:}

Prevención Universal, Conductas Alimentarias Saludables, Autoeficacia, Afecto Positivo, Autoatribución.

\footnotetext{
a Autor de Correspondencia, Universidad Autónoma del Estado de Hidalgo, Instituto de Ciencias de la Salud (ICSA), San Agustín Tlaxiaca, Hgo., México, https://orcid.org/00000002-1427-3697, Email: anahi_gaspar@uaeh.edu.mx

${ }^{\mathrm{b}}$ Universidad Autónoma del Estado de Hidalgo, Instituto de Ciencias de la Salud (ICSA), San Agustín Tlaxiaca, Hgo., México, https://orcid.org/0000-0003-0877-4871, Email: remar64@yahoo.com.mx

¿ Universidad Autónoma del Estado de Hidalgo, Instituto de Ciencias de la Salud (ICSA), San Agustín Tlaxiaca, Hgo., México, https://orcid.org/0000-0002-3969-683X, Email: Lilian_bosques@uaeh.edu.mx

${ }^{\mathrm{d}}$ Universidad Nacional Autónoma de México, Facultad de Psicología, Ciudad de México, México, https://orcid.org/0000-0001-9878-3653, Email: romsip@unam.mx Universidad Nacional Autónoma de México, Facultad de Estudios Superiores Iztacala, Ciudad de México, México https://orcid.org/0000-0001-6491-9639 Email: rvamap@unam.mx

Universidad Autónoma del Estado de México, Centro de Investigación en Ciencias Médicas, Toluca de Lerdo, México https://orcid.org/0000-0001-5901-3787,
} 


\section{Introducción}

De acuerdo con la Organización Mundial de la Salud (OMS), la salud está ligada a dos factores determinantes; la alimentación y la actividad física. Para que una persona se mantenga sana es necesario que consuma alimentos de forma equilibrada [1], permitiéndole un adecuado desarrollo de las capacidades físicas e intelectuales [2].

La Conducta Alimentaria (CA) es un conjunto de acciones realizadas por el individuo en respuesta a una motivación biológica, psicológica y sociocultural, relacionada con la ingestión de alimentos [3]. Así, el comportamiento alimentario influye en gran medida en el estado de salud, especialmente en niños y adolescentes, pues en esta etapa la alimentación saludable depende de hábitos y comportamientos alimenticios adecuados [4], además, la adolescencia es un periodo de importantes cambios emocionales, sociales y cognitivos, por lo que la adquisición de ciertos hábitos poco saludables puede comprometer su salud [5].

Otro aspecto importante es que, los estados nutricionales fuera del normopeso representan un grave problema de salud pública en el mundo. México ocupa el primer lugar en obesidad infantil a nivel mundial [6], el $36.3 \%$ de los adolescentes presentan sobrepeso y obesidad, mientras que, en el otro extremo, el $5.6 \%$ de los adolescentes presenta anemia [7].

Desde la Psicología, un factor identificado de gran importancia para el cuidado de la salud es la autoeficacia, pues está relacionada con el desarrollo de conductas favorables para el cuidado y mantenimiento de la salud, e influye en la respuesta emocional de una personal en relación con su percepción de bienestar, vitalidad y actitud ante el cambio de conductas saludables [8]. Otro factor asociado con el desarrollo hábitos saludables es el afecto positivo, pues si se desarrolla durante la adolescencia puede generar experiencias positivas y sanas en la vida adulta [9]. De igual manera, al autoatribuirse aspectos subjetivos positivos, existe mayor probabilidad de que el adolescente tenga una imagen más positiva de sí mismo y del cuidado de su salud [10].

Gillespie encontró en su investigación que los niveles más altos de autoeficacia están asociados con un mayor consumo de fruta, y mejora la selección de alimentos y bebidas azucaradas. También encontró que, los participantes con altos niveles de autoeficacia mostraron una capacidad estadísticamente significativa para consumir vegetales [11].

Por otro lado, Esparza y Rodríguez reportaron en su estudio que los trastornos de ansiedad y del estado de ánimo son los más comunes en los adolescentes [12], y Gairagarboil, encontró que el autoconcepto y la percepción del ambiente influyen en el balance emocional del individuo, ya sea positivamente (alta autoestima) o negativamente (baja autoestima), afectando de esta manera el comportamiento del adolescente [13].

Por lo tanto, la promoción y prevención de la salud han sido utilizadas como estrategias para desarrollar diversas intervenciones dirigidas a personas $y$ comunidades [14], en los desórdenes de alimentación se ha encontrado que estas estrategias minimizan el riesgo de su aparición [15] y contribuyen además, en la adopción de estilos de vida saludables en la adolescencia. Los estudios nacionales e internacionales basados en prevención universal para Conductas Alimentarias de Riesgo (CAR) y en promoción de Conductas Alimentarias Saludables están dirigidos a hombres y mujeres de primaria y secundaria [16-20], e incluyen además de la intervención en variables psicológicas, el desarrollo de la alimentación saludable y la actividad física, obteniendo resultados favorables en la modificación de dichas conductas.

Cabe resaltar que, el estilo de vida puede ser modificado y reforzado desde la escuela, donde las estrategias educativas deben dirigirse a desarrollar habilidades y aumentar la sensibilización sobre el cuidado de la salud en los estudiantes [21].

Por lo tanto, el objetivo de este estudio fue evaluar la efectividad de un programa de prevención universal basado en autoeficacia, afecto positivo y autoatribución para promover Conductas Alimentarias Saludables en escolares de educación secundaria de primer y segundo grado.

\section{Materiales y Métodos}

\section{Tipo de estudio y diseño experimental}

El estudio fue cuasi experimental con cuatro grupos: dos grupos experimentales y dos grupos en fase de espera; con pre y post evaluación, donde de manera aleatoria se asignaros a los participantes de acuerdo al grado y grupo al que pertenecían a los grupos experimentales; y lo mismo a los grupos en fase de espera.

\section{Participantes}

La muestra estuvo conformada por $\mathrm{N}=129$ adolescentes, de los cuales $n=63$ pertenecieron a los grupos experimentales y $n=66$ a los grupos en fase de espera. De la muestra total $n=68(53 \%)$ son mujeres y $n=61$ (47\%) hombres, teniendo un rango de edad de 12 a 15 años, $\quad \mu=13$ años $y \quad D E=.79$. 


\section{Instrumentos}

Inventario de Autoeficacia para el control de peso: tiene un alfa de Cronbach de .88, consta de 20 ítems con escala tipo Likert donde las opciones de respuesta van de "No soy capaz de hacerlo" (1) a "Soy muy capaz de hacerlo" (4) [22]. Consta de dos factores; autoeficacia para la ingesta de alimentos (8 ítems) y autoeficacia para la actividad física (12 ítems).

Escala de afecto positivo y negativo (PANAS): obtuvo un alfa de Cronbach de 0.82, consta de 18 items con opciones de respuesta en una escala Likert que va desde "Nunca" (1) hasta "Siempre" (5), esta escala considera dos grandes factores para evaluar las experiencias emocionales; Afecto positivo (AP) con 8 items y afecto negativo (AN) con 10 items [23].

Inventario de Autoatribución para adolescentes de secundaria: tiene un alfa de Cronbach de 0.83. Consta de 33 items con opciones de respuesta en una escala Likert que va desde "No me describe nada" (1) hasta "Me describe exactamente" (5), y se agrupa en dos factores; autoatribución positiva con 17 items y autoatribución positiva con 12 items [24].

\section{Procedimiento}

Se acudió con las autoridades de una secundaria pública de Mineral de la Reforma, Hidalgo para darles a conocer el proyecto. Una vez que los directivos dieron su aprobación, se obtuvo el consentimiento informado de los padres de familia. Al llegar a la secundaria, la subdirectora proporcionó los grupos que participarían en este estudio. Al pasar a los salones, se les explicó a los participantes el objetivo de la intervención y se les pidió su consentimiento, de igual manera, se les explicó que al ser su participación voluntaria podrían retirarse del estudio cuando lo decidieran sin ninguna consecuencia. Posteriormente, completaron la batería de pruebas dentro del horario de clase en el siguiente orden: Escala de Afecto Positivo y Negativo (PANAS), Inventario de Autoeficacia para el control de peso e Inventario de Autoatribución para adolescentes de secundaria. Los grupos experimentales iniciaron su intervención después de la primera evaluación, mientras los grupos en fase de espera siguieron con sus clases habituales.

\section{Propuesta del Programa de Intervención}

El presente programa de intervención tuvo como propósito promover la adquisición de estilos saludables de alimentación y de actividad física, el desarrollo de habilidades como la autoeficacia (reforzada con técnicas de disonancia cognoscitiva) para afrontar adecuadamente factores de riesgo de CAR, así como el manejo saludable del afecto negativo (estrés, ansiedad y depresión) e incrementar la autoatribución positiva. El programa psicoeducativo consistió en 3 sesiones de 50 minutos cada una.

\section{Análisis estadístico}

El análisis de datos se llevó a cabo con el paquete estadístico SPSS versión 21 para Windows. Para determinar la equivalencia inicial entre los grupos experimentales y los grupos en fase de espera se utilizó la prueba estadística $t$ de student para muestras independientes, mientras que para determinar el efecto del programa de intervención se utilizó la prueba estadística $t$ de student para muestras relacionadas.

\section{Resultados}

En primer lugar, se describen las características generales de la muestra:

Como se aprecia en la figura 1 , el $50.39 \%$ de los participantes se encuentran preocupados por su peso corporal, mientras que el $49.61 \%$ de ellos no lo están. Respecto a los participantes que han realizado dieta restrictiva (porciones pequeñas, dejar de comer algunos alimentos), el $37.21 \%$ afirman haberlo hecho, mientras que la mayoría de ellos $62.79 \%$ no han realizado una dieta restrictiva (figura 2). En cuanto la percepción de la figura corporal, la figura 3 refleja que el $6.20 \%$ de los participantes creen estar muy delgados, el $29.46 \%$ delgados, el $53.49 \%$ ni gordo/as ni delgado/as, el $9.30 \%$ de los participantes creen estar gordo/as y el1.55\% muy gordo/as. 
Figura 1. Distribución porcentual de la preocupación por el peso corporal en la muestra

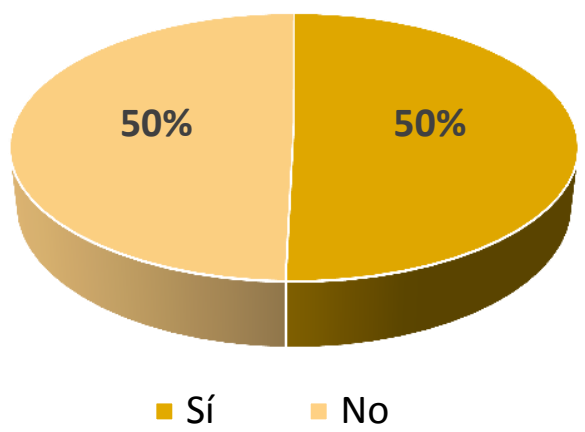

Figura 2. Distribución porcentual de la muestra en haber realizado dieta restrictiva (porciones pequeñas, dejar de comer algunos alimentos)

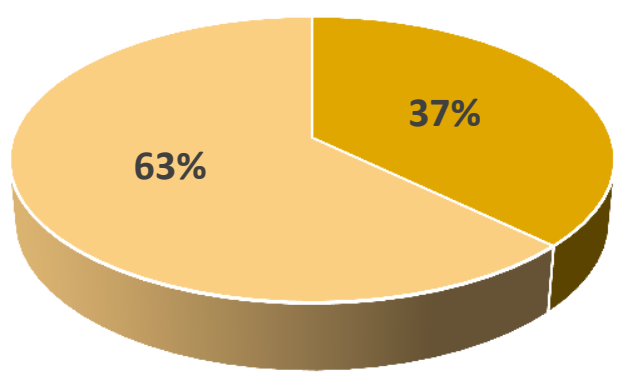

- Sí $\square$ No

Figura3. Distribución porcentual de la muestra sobre la percepción de la figura corporal.

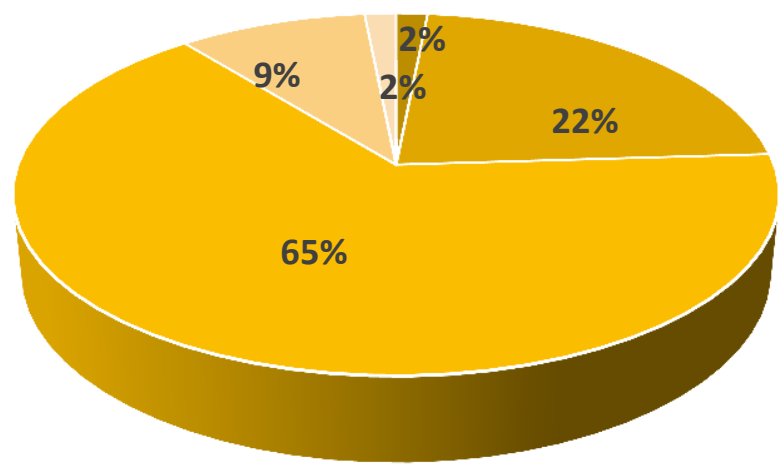

- Muy delgado/a $=$ Delgado/a
- Ni gordo/a ni delgado/a - Gordo/a
" Muy gordo/a

Posteriormente, se llevó a cabo una comparación entre las puntuaciones obtenidas en los instrumentos que fueron aplicados durante la pre-evaluación; este análisis se llevó a cabo mediante la prueba estadística $t$ de student para muestras independientes. En las puntuaciones obtenidas, no se observaron diferencias estadísticamente significativas entre el afecto, la autoeficacia y la autoatribución entre los grupos. Por lo tanto, se estableció que existe equivalencia entre las condiciones antes de la intervención (ver tabla 1).

Tabla 1. Equivalencia inicial entre grupos experimentales $y$ en fase de espera.

\begin{tabular}{lcccc}
\hline & $\begin{array}{c}\text { Grupos } \\
\text { experimentales }\end{array}$ & $\begin{array}{c}\text { Grupos } \\
\text { en fase } \\
\text { de espera }\end{array}$ & & \\
\hline Escala/ medición & Media & Media & Valor t & $\begin{array}{c}\text { Valor } \\
\text { de p }\end{array}$ \\
Afecto: & 1.81 & 2.19 & 3.12 & .53 \\
Negativo & 3.54 & 3.38 & 1.00 & .21 \\
Positivo & & & & .80 \\
Autoatribución: & 2.20 & 2.55 & 3.04 & .80 \\
Negativa & 3.67 & 3.28 & 3.92 & .37 \\
Positiva & & & & \\
Autoeficacia para: & 3.21 & 3.08 & 1.54 & .95 \\
Ingesta de alimentos & 2.94 & 2.80 & .180 & .16 \\
Actividad física & & & & \\
\hline
\end{tabular}

${ }^{*} p \leq .05$ 


\section{Hidalgo, Vol.8, No. 15 (2019) 24-30}

Finalmente, para determinar el impacto del programa de prevención universal se utilizó la prueba $t$ de student para muestras relacionadas. Para los análisis se incluyeron a los cuatro grupos de estudio: a) grupo experimental de primer grado, b) grupo experimental de segundo grado, c) grupo en fase de espera de primer grado y d) grupo en fase de espera de segundo grado; cada uno con dos mediciones: pre test y post test.

A continuación, se describen estos resultados:

Tabla 2. Diferencias entre pretest y postest en Afecto positivo y negativo, Autoeficacia para la ingesta de alimentos y para la actividad física y Autoatribución positiva y negativa, en los grupos experimentales.

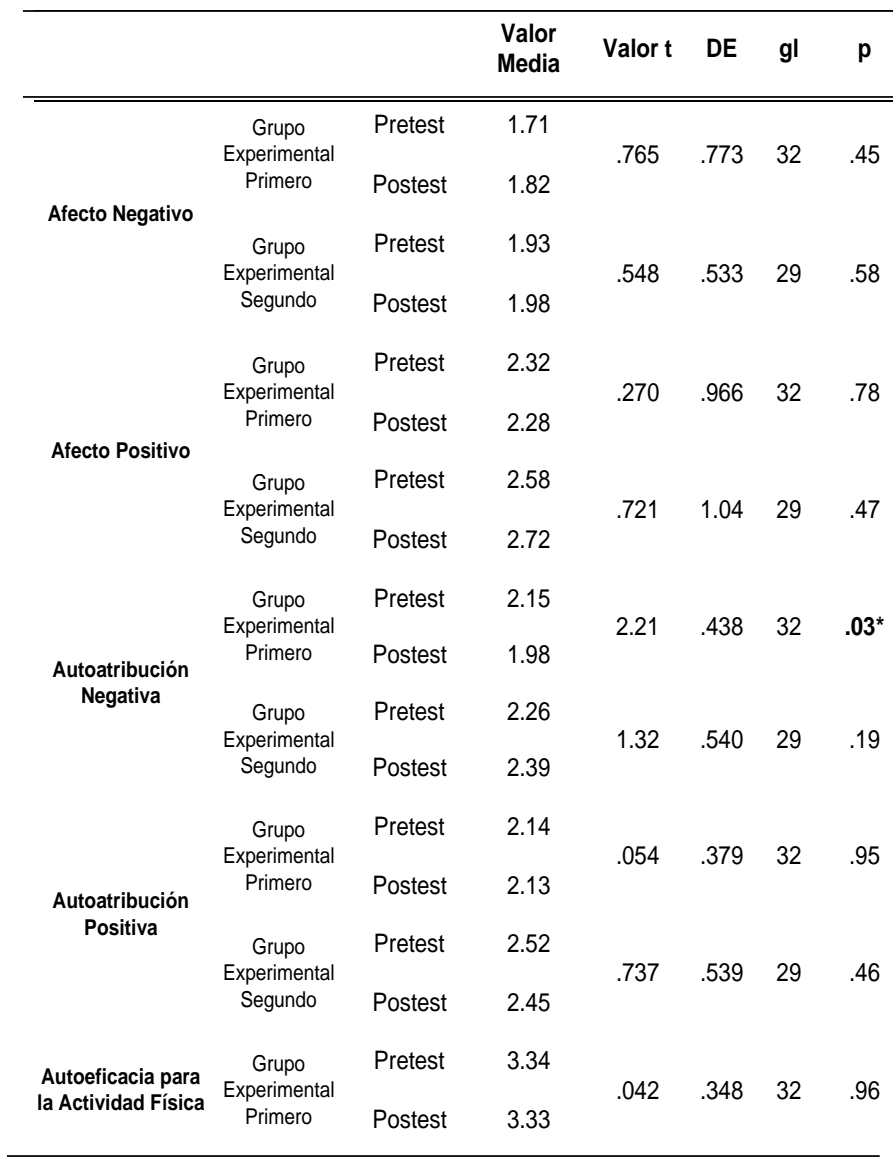

${ }^{*} p \leq .05$

En la tabla 2, se observan diferencias estadísticamente significativas en el nivel de autoatribución negativa en el grupo experimental de primer año antes y después de la intervención, $t \quad(32)=2.21, p<0.03$, disminuyendo significativamente la autoatribución negativa $(\mu=2.15)$ después de la intervención $(\mu=1.98)$.

En los grupos en fase de espera, la tabla 3 muestra que se encontraron diferencias estadísticamente significativas en el nivel de autoatribución positiva en el grupo en fase de espera de segundo año, $\mathrm{t}(34)=2.27, \mathrm{p}<0.02$, disminuyendo significativamente la autoatribución positiva $(\mu=2.70)$ en el postest $(\mu=2.54)$. Así mismo, se encontraron diferencias estadísticamente significativas en el nivel de autoeficacia para la actividad física del grupo en fase de espera de primer año $\mathrm{t}(30)=2.01, \mathrm{p}<.05$, disminuyendo significativamente la autoeficacia para la actividad física $(\mu=3.02)$ en el postest $(\mu=2.71)$.

Tabla 3. Diferencias entre pretest y postest en Afecto positivo y negativo, Autoeficacia para la ingesta de alimentos y para la actividad física y Autoatribución en los grupos en fase de espera

\begin{tabular}{|c|c|c|c|c|c|c|c|}
\hline & & & $\begin{array}{l}\text { Valor } \\
\text { Media }\end{array}$ & $\underset{t}{\text { Valor }}$ & $\mathrm{DE}$ & gl & $\mathbf{p}$ \\
\hline \multirow{2}{*}{ Afecto Negativo } & $\begin{array}{l}\text { Grupo en fase de } \\
\text { espera Primero }\end{array}$ & $\begin{array}{l}\text { Pretest } \\
\text { Postest }\end{array}$ & $\begin{array}{l}2.11 \\
2.04\end{array}$ & .588 & .641 & 30 & .56 \\
\hline & $\begin{array}{l}\text { Grupo en fase de } \\
\text { espera Segundo }\end{array}$ & $\begin{array}{l}\text { Pretest } \\
\text { Postest }\end{array}$ & $\begin{array}{l}2.26 \\
2.24\end{array}$ & .298 & .397 & 34 & .76 \\
\hline \multirow{2}{*}{ Afecto Positivo } & $\begin{array}{l}\text { Grupo en fase de } \\
\text { espera Primero }\end{array}$ & $\begin{array}{l}\text { Pretest } \\
\text { Postest }\end{array}$ & $\begin{array}{l}2.60 \\
2.64\end{array}$ & .210 & .853 & 30 & .83 \\
\hline & $\begin{array}{l}\text { Grupo en fase de } \\
\text { espera Segundo }\end{array}$ & $\begin{array}{l}\text { Pretest } \\
\text { Postest }\end{array}$ & $\begin{array}{l}2.62 \\
2.55\end{array}$ & .701 & .627 & 34 & .48 \\
\hline \multirow{2}{*}{$\begin{array}{l}\text { Autoatribución } \\
\text { Negativa }\end{array}$} & $\begin{array}{l}\text { Grupo en fase de } \\
\text { espera Primero }\end{array}$ & $\begin{array}{l}\text { Pretest } \\
\text { Postest }\end{array}$ & $\begin{array}{l}2.45 \\
2.32\end{array}$ & 1.25 & .594 & 30 & .21 \\
\hline & $\begin{array}{l}\text { Grupo en fase de } \\
\text { espera Segundo }\end{array}$ & $\begin{array}{l}\text { Pretest } \\
\text { Postest }\end{array}$ & $\begin{array}{l}2.64 \\
2.52\end{array}$ & 1.23 & .594 & 34 & .22 \\
\hline \multirow{2}{*}{$\begin{array}{c}\text { Autoatribución } \\
\text { Positiva }\end{array}$} & $\begin{array}{l}\text { Grupo en fase de } \\
\text { espera Primero }\end{array}$ & $\begin{array}{l}\text { Pretest } \\
\text { Postest }\end{array}$ & $\begin{array}{l}2.71 \\
2.80\end{array}$ & .850 & .584 & 30 & .40 \\
\hline & $\begin{array}{l}\text { Grupo en fase de } \\
\text { espera Segundo }\end{array}$ & $\begin{array}{l}\text { Pretest } \\
\text { Postest }\end{array}$ & $\begin{array}{l}2.70 \\
2.54\end{array}$ & 2.27 & .433 & 34 & $.02^{\star}$ \\
\hline Autoeficacia para la & $\begin{array}{l}\text { Grupo en fase de } \\
\text { espera Primero }\end{array}$ & $\begin{array}{l}\text { Pretest } \\
\text { Postest }\end{array}$ & $\begin{array}{l}3.02 \\
2.71\end{array}$ & 2.01 & .855 & 30 & $.05^{\star}$ \\
\hline$A C$ & $\begin{array}{l}\text { Grupo en fase de } \\
\text { espera Segundo }\end{array}$ & $\begin{array}{l}\text { Pretest } \\
\text { Postest }\end{array}$ & $\begin{array}{l}3.14 \\
3.09\end{array}$ & .679 & .435 & 34 & .50 \\
\hline
\end{tabular}

${ }^{*} p \leq .05$

\section{Discusión}

Esta investigación cumplió con el objetivo de evaluar la efectividad de un programa basado en prevención universal sobre autoeficacia, afecto positivo y autoatribución para promover conductas alimentarias saludables en escolares de educación secundaria, comparando resultados de dos momentos de evaluación: pretest y postest.

El programa resultó ser relativamente adecuado, pues modificó la variable autoatribución negativa en el grupo experimental de primer año. Sin embargo, no mostró tener un impacto significativo en la modificación de los niveles de autoeficacia para la ingesta de alimentos, autoeficacia para la actividad física, afecto positivo, afecto negativo y autoatribución positiva.

El grupo experimental de primer año presentó cambios significativos en la disminución de los niveles de 


\section{Hidalgo, Vol.8, No. 15 (2019) 24-30}

autoatribución negativa después de la intervención. Este resultado es de gran importancia, pues reducir la autoatribución negativa resulta ser una experiencia positiva para el adolescente, ya que la baja autoestima que es un componente de esta variable, se encuentra presente en las personas diagnosticadas con trastornos de la alimentación [25]. En un estudio realizado por Ramos-Díaz y otros en 1250 estudiantes de secundaria en España, se demostró que los adolescentes con un autoconcepto alto están más satisfechos con la vida y presentan mayor afecto positivo, en comparación con aquellos con un autoconcepto bajo quienes además muestran la presencia de afecto negativo [26].

Por otro lado, el grupo en fase de espera de segundo año mostró cambios significativos en la disminución del nivel de autoatribución positiva en el postest. Al respecto, Crocker y colaboradores, señalan que los incrementos y decrementos en la autoestima de los adolescentes dependerá de los éxitos o fracasos que experimenten en la vida, así como de las circunstancias en que se presenten [27].

Respecto a la variable afecto, no se encontraron cambios estadísticamente significativos en el nivel de afecto negativo y afecto positivo tanto en los grupos experimentales como en los grupos en fase de espera. Estos resultados se relacionan con lo que algunos estudios sugieren sobre la necesidad de un mayor desarrollo de programas dirigidos a la prevención e intervención para disminuir el efecto negativo en esta población, y para tener una mejor comprensión de estrategias que mejoren el estado de ánimo de niños y adolescentes, así como la eficacia de estos programas [28].

En los resultados de la autoeficacia para la actividad física en los grupos en la fase de espera, el grupo de primero tuvo cambios significativos, disminuyendo el nivel de esta variable en el postest, ante estos resultados algunos estudios reportan que las mayores disminuciones en la actividad física se presentan en la adolescencia, siendo una condición normal, pues los adolescentes tienden a volverse más sedentarios en esta etapa de desarrollo [29], lo cual también influye para que en los participantes de los grupos experimentales no se reportaran cambios significativos.

\section{Conclusión}

Dentro de las principales aportaciones de esta investigación está el haber desarrollado un programa breve que siente las bases de la variable autoatribución, pues ha sido poco estudiada en México y sobre todo en el estado de Hidalgo, mostrando ser efectiva esta intervención para disminuir la autoatribución negativa en escolares de secundaria. Aunque no se reportaron cambios en la autoatribución positiva el disminuir la negativa impacta directamente el autoconcepto y refuerza los atributos positivos de los estudiantes.

Es importante seguir aportando programas de intervención breves basados en prevención universal, ya que son de bajo costo y pueden ser aplicados a un gran número de población, además de dar resultados efectivos a corto plazo. Estos programas deben ser acordes a las características y a la etapa de desarrollo de la población a intervenir, además de considerar los factores psicológicos y socioculturales vinculados a los problemas de alimentación.

Por lo anterior, se sugiere la réplica de este programa, desarrollando cada una de las variables por separado para identificar los contenidos con mayor efectividad, y posteriormente unir estos contenidos en el programa. Además, se sugiere hacer un seguimiento a nueve meses para medir la permanencia de los cambios en el tiempo.

Es importante señalar que, una de las principales limitaciones de este estudio es el carácter no probabilístico de la muestra, lo que impide la generalización de los resultados obtenidos a la población adolescente mexicana.

\section{Agradecimientos}

El presente artículo fue realizado gracias al apoyo recibido por parte del Consejo Nacional de Ciencia y Tecnología (CONACyT), con la beca número 627660 para la realización de estudios de posgrado en el programa de Maestría en Ciencias Biomédicas y de la Salud de la Universidad Autónoma del Estado de Hidalgo.

\section{Referencias}

[1] Organización Mundial de la Salud. Alimentación Sana [Internet]. 31 de agosto de 2018. 2018 [citado el 5 de enero de 2019]. Disponible en: https://www.who.int/es/news-room/fact-sheets/detail/healthy-diet

[2] Ricardo YR, Peralta L, Yaulema L, Pallo J, Caiza V, Parreño Á, et al. Alimentación saludable en docentes. Rev Cuba Med Gen Integr. 2017;33(1):115-28.

[3] Lora-Cortez, CI; Saucedo-Molina T. Conductas alimentarias de riesgo e imagen corporal de acuerdo al índice de masa corporal en una muestra de mujeres adultas de la ciudad de México. alud Ment. 2006;29(3):60-7.

[4] Castells M; Capdevila C; Girbau T; Rodríguez C. Estudio del comportamiento alimentario en escolares de 11 a 13 años de Barcelona. Nutr Hosp. 2006;21(4):517-32.

[5] Garc M, Castro MM. Hábitos de vida saludable desde la adolescencia. Rev estidios Juv. 2016;112(2):107-16.

[6] Salud y Nutricion. Fondo de las Naciones Unidas para la Infancia [Internet]. 2018 [citado el 27 de mayo de 2019]. Disponible en: https://www.unicef.org/mexico/spanish/17047_17494.html

[7] Instituto Nacional de Salud Pública. Encuesta Nacional de Salud y Nutrición de Medio Camino 2016 Informe Final de Resultados 
[Internet]. 2016. Disponible en: http://oment.uanl.mx/wpcontent/uploads/2016/12/ensanut_mc_2016-310oct.pdf

[8] Schonfeld P; Brailovskaia J; Bieda A; Chi-Zhang X; Margraf J. The effects of daily stress on positive and negative mental health: mediation through self-efficacy. Int J Clin Heal Psychol. 2016;16:110.

[9] Yıldız MA, Kızıldağ S. Pathways from Positive and Negative Affect to Depressive Symptoms: Multiple Mediation of Emotion Regulation Strategies. 2018;34(2013):241-50.

[10] Myer D. Psicología Social. México: Mc Graw Hill; 1995.

[11] Gillespie R. Connecting self-efficacy of dietary choices and the association with dietary intake among rural adolescents in NorthCcarolina and Kentucky. Diet Hum Nutr. 2017;54:1-27.

[12] Esparza N; Rodríguez M. Factores contextúales del desarrollo infantil y su relación con los estados de ansiedad y depresión. Divers Perspect en Psicol. 2009;5(1):47-64.

[13] Garaigordobil M, Oñederra J. Inteligencia emocional en las víctimas de acoso escolar y en los agresores. Eur J Educ Psychol. 2010;3(2):243-56.

[14] Pettersen G, Rosenvinge JH, Wynn R. Eating disorders and psychoeducation - patients ' experiences of healing processes. 2011;(12):12-9.

[15] Belak L, Deliberto T, Shear M, Kerrigan S, Attia E. Inviting eating disorder patients to discuss the academic literature : a model program for psychoeducation. 2017;1-7.

[16] Varnado-Sullivan PJ, Horton R. Acceptability of programs for the prevention of eating disorders. J Clin Psychol. 2006;62:687-703.

[17] Stice E; Presnell K. The body Project: Promoting body acceptance and preventing eating disorders. Oxford, editor. New York; 2007.

[18]Black C, Mcdaniel L, Bull S, Powell M, Mcintyre K. Can we reduce eating disorder risk factors in female college athletes? A randomized exploratory investigation of two peer-led interventions $\longleftarrow$. Body Image. 2012;9(1):31-42.

[19] Berger U, Schaefer J, Wick K, Brix C. Effectiveness of Reducing the Risk of Eating-Related Problems Using the German School-Based Intervention Program, "Torera ", for Preadolescent Boys and Girls. Prev Sc. 2013;(1-11).

[20]Soto D. Intervención Psicoeducativa para Prevenir Factores Psicosociales asociados a Actitudes y Conductas Alimentarias No Saludables en Escolares. 2014.

[21] Galván M, López G, Gúzman R, Fernández TL, Atitlán A, Vargaz E, Amezcua A, Hernández J. Educación en Salud y Nutrición en Escuelas Primarias: Los Proyectos Formativos Como Estrategia Innovadora de Planificación Didáctica . 2016.

[22] Gómez-Peresmitré G, Platas S, Pineda-García G, Guzmán- Salda R, León-Hernandez R. Validation of an instrument of self-efficacy for the prevention of obesity in school children. Rev Mex Trastor Aliment. 2017;8:21-30.

[23] Gómez-Peresmitré G, Platas S, Pineda-García G, Corona N, González V. Propiedades psicométricas de la Escala de Afecto negativo y Afecto positivo (PANAS) en adolescentes de secundaria. La Psicol Soc en México. 2018;17.

[24] Gómez-Peresmitré G, Platas S, Pineda-García G, Corona N, González V. Propiedades psicométricas del inventario de autoatribución para adolescentes de secundaria. La Psicol Soc en México. 2018;17.

[25] Moreno MOG. Trastornos Alimentariosy su relación con la Imagen Corporal y la Autoestima en Adolescentes. Ter Psicol. 2009;27(2):181-90.

[26] Ramos-Díaz E; Rodríguez-Fernández A; Antonio-Aguirre I. El autoconcepto y el bienestar subjetivo en función del sexo y del nivel educativo en la adolescencia. Psicol Educ. 2017;23:89-94.
[27] Crocker J, Sommers SR, Luhtanen R. Hopes dashed and dreams Fulfilled: Contingencies of self-worth and graduate school admissions. Personal Soc Psychol Bull. 2002;28(1275-1286).

[28] McCabe MP, Ricciardelli LA, Salmon JO. Evaluation of a prevention program to address body focus and negative affect among children. $\mathrm{J}$ Health Psychol. 2006;11(4):589-98.

[29] Muros JJ, Ocaña-lara FA. Results of a 7-Week School-Based Physical Activity and Nutrition Pilot Program on Health-Related Parameters in Primary School Children in Southern Spain. 2013;24861. 